\title{
WEIGHTS AND MEASURES
}

On 1 Jan. 1960 following an agreement between the standards laboratories of Great Britain, Canada, Australia, New Zealand, South Africa and the USA, an international yard and an intemational pound (avoirdupois) came into existence. 1 yard $=91.44$ centimetres; $1 \mathrm{lb} .=453.59237$ grammes .

The abbreviation ' $\mathrm{m}$ ' signifies 'million(s)' and tonnes implies metric tons.

\begin{tabular}{|c|c|c|c|}
\hline \multicolumn{2}{|c|}{ LENGTH } & \multicolumn{2}{|c|}{ DRY MEASURE } \\
\hline $\begin{array}{l}\text { Centimetre } \\
\text { Metre } \\
\text { Kilometre }\end{array}$ & $\begin{array}{l}0.394 \text { inch } \\
1.094 \text { yards } \\
0.621 \text { mile }\end{array}$ & $\begin{array}{l}\text { Litre } \\
\text { Hectolitre }\end{array}$ & $\begin{array}{l}0.91 \text { quart } \\
2.75 \text { bushels }\end{array}$ \\
\hline \multicolumn{2}{|c|}{ LIQUID MEASURE } & \multicolumn{2}{|c|}{ WEIGHT-AVOIRDUPOIS } \\
\hline \multirow{3}{*}{$\begin{array}{l}\text { Litre } \\
\text { Hectolitre }\end{array}$} & \multirow{3}{*}{$\begin{array}{l}1.75 \text { pints } \\
22 \text { gallons }\end{array}$} & \multirow{3}{*}{$\begin{array}{l}\text { Gramme } \\
\text { Kilogramme } \\
\text { Quintal ( = } \\
100 \mathrm{~kg}) \\
\text { Tonne (= } \\
1,000 \mathrm{~kg})\end{array}$} & 15.42 grains \\
\hline & & & $2 \cdot 205$ pounds \\
\hline & & & $\begin{array}{l}220.46 \text { pounds } \\
0.984 \text { long ton } \\
1.102 \text { short tons }\end{array}$ \\
\hline \multicolumn{2}{|c|}{ SURFACE MEASURE } & \multirow{2}{*}{\multicolumn{2}{|c|}{ WEIGHT-TROY }} \\
\hline & $10.76 \mathrm{sg}$ feet & & \\
\hline Hectare & 2.47 acres & Kilogramme & $32 \cdot 15$ ounces \\
\hline Square kilometre & 0.386 sq. mile & ninogramme & 2.68 pounds \\
\hline
\end{tabular}

\section{BRITISH WEIGHTS AND MEASURES}

\begin{tabular}{|c|c|c|c|}
\hline \multicolumn{2}{|c|}{ LENGTH } & \multicolumn{2}{|r|}{ WEIGHT } \\
\hline $\begin{array}{l}1 \text { foot } \\
1 \text { yard } \\
1 \text { mile }(= \\
1,760 \text { yds })\end{array}$ & $\begin{array}{l}0.305 \text { metre } \\
0.914 \text { metre } \\
1.609 \text { kilometres }\end{array}$ & $\begin{array}{l}1 \text { ounce }(= \\
437 \cdot 2 \text { grains }) \\
1 \mathrm{lb}(=7,000 \\
\text { grains) } \\
1 \text { cwt. }(=112 \\
\text { lb. } \\
1 \text { long ton (= } \\
2,240 \mathrm{lb} .) \\
1 \text { short ton (= } \\
2,000 \mathrm{lb} .)\end{array}$ & $\begin{array}{c}28.350 \text { grammes } \\
453.6 \text { grammes } \\
\begin{array}{c}50.802 \text { kilo- } \\
\text { grammes }\end{array} \\
1.016 \text { tonnes } \\
0.907 \text { tonne }\end{array}$ \\
\hline \multicolumn{2}{|c|}{ SURFACE MEASURE } & \multicolumn{2}{|c|}{ LIQUID MEASURE } \\
\hline $\begin{array}{l}\text { I sq. foot } \\
\text { l sq. yard } \\
\text { I acre } \\
\text { I sq. mile }\end{array}$ & $\begin{array}{l}9.290 \text { sq. decimetres } \\
0.836 \text { sq. metre } \\
0.405 \text { hectare } \\
2.590 \text { sq. kilometres }\end{array}$ & $\begin{array}{l}1 \text { pint } \\
1 \text { gallon } \\
1 \text { quarter }\end{array}$ & $\begin{array}{l}0.568 \text { litre } \\
4.546 \text { litres } \\
2.909 \text { hectolitres }\end{array}$ \\
\hline
\end{tabular}

